\title{
Design of Robot Arm imitating Human Arm system using PID Controller
}

\author{
Pan Myat Phyu \\ Department of Electronic \\ Engineering \\ Technological University \\ Thanlyin, Myanmar
}

\author{
Dr. Yin Yin Soe \\ Department of Electronic \\ Engineering \\ Technological University \\ Thanlyin, Myanmar
}

\author{
Daw May Thu Aung \\ Department of Electronic \\ Engineering \\ Technological University \\ Thanlyin, Myanmar
}

\begin{abstract}
The purpose system is to connect humans and robots in order to reproduce operator action at a distance. An inexpensive, a lightweight and easily controlled robotic arm with human interface is developed. The developed robotic arm is fully functional and accurately reacts to the movements from the human arm. The purpose system consist of a 'master', a human arm, and a 'slave', robot arm, located at a remote site. The human arm is given position force and the slave receives a position reference from the human. The human user wears 2 sensors on his/her arm. These sensors relay position information to the robotic arm. This can be achieved with the help of simple Gyro sensor at the joints of the user arm. The Position-force is designed and implemented using a PID controller. PID controllers are most popular and most often used controllers in industry. Popularity of the PID controllers are due to their wide range of operating conditions and functional simplicity. Different types of tuning rules have proposed which can fine tune the system to get desired response. In this, manual tuning method of PID controller is used to control of position of DC motor using Arduino microcontroller. Overall aim is to make the microcontroller understand human body language, thereby bridging the gap between machine and human. This thesis presents a PID controller method by using MATLAB R2017a simulation and experiment result to accurate robot action is similar to human arm action. According to the result, the robot action is similar human arm action to $98 \%$.
\end{abstract}

Keywords: gyro sensor; Arduino; PID controller; master; slave.

\section{INTRODUCTION}

As technology becomes more advanced, machines become more complex and intelligent. Now a days, robots are able to carry heavy objects and operate with a precision which is below the range of human hand . Other applications again require the operation in human-unfriendly or hazardous environments. Thus, at present, typical application fields for robots can be found in areas where human physical abilities are exceeded or the usage in human-inaccessible or unfriendly environments is necessary.

In technical terms a system consists of a human arm is embedded Gyro sensor and a slave robot arm and a communication channel which controls the transfer position information. The angle can be read easily with the help of a sensors embedded human arm and this angle is transmitted to the robot arm [1].

This technique is very useful since it takes real time of human arm and tracks it to get interface with robotic arm. Tracking of such hand will interface the controller with robotic arm. The main aim behind this approach to program a robotic arm, so that it should be controlled by human hand and will reach the locations where human will not be able to reach and do the given task by direct interfacing with human arm. In this we can see the real time movement of robotic arm [2].The hardware setup consists of two Pololu-75:1 Metal Gearmotor 37D $\times 52 \mathrm{~L} \mathrm{~mm}$ with $48 \mathrm{CPR}$ Encoder ,two Gyro sensors and Arduino Mega board .Gyro sensors is embedded in human arm and sensors are read human arm position and then control the robot arm to same human arm action using PID controller.

The implementation of a proportional integral derivative (PID) controller was used in this system. They controlled the position of the DC motors with pulse width modulation using Arduino Mega Board. This system is shown in Figure 1.

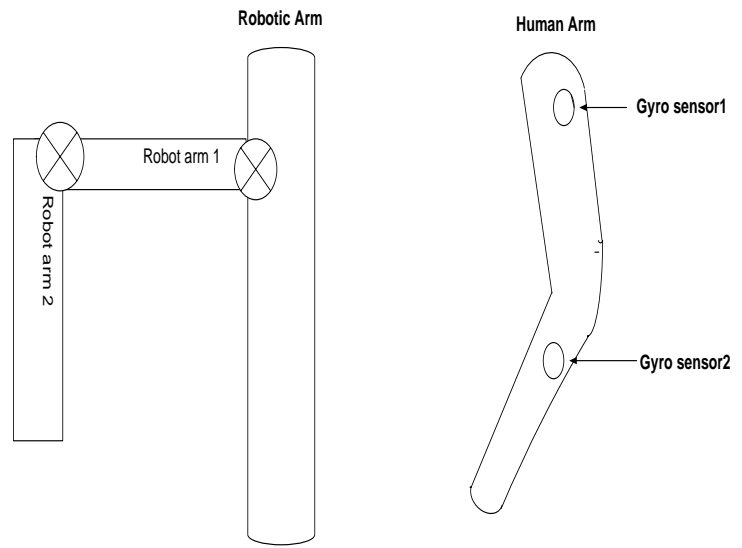

Figure 1. The Diagram of the Purpose System

\section{RELATED WORK}

\subsection{Arduino Mega Board}

The microcontroller board used in the robotic arm system is Arduino Mega, which is shown in Figure 2. The board is sufficient for our design, and there are enough input and output pins for the project. 


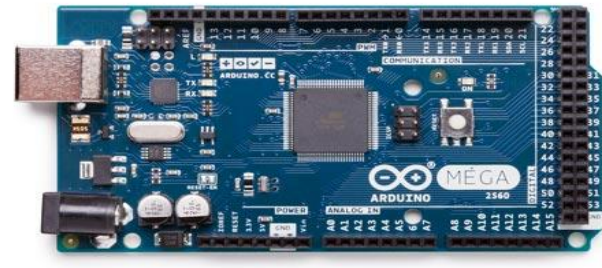

Figure 2. Arduino Mega Board

\subsection{Gyro Sensor}

This IMU 10DOF is a motion tracking module. Its design is based on the sensor MPU6050,HMC5883L and BMP085.The sensor MPU6050 which is the world's first integrated 6-axis Motion Tracking device, that combines a 3-axis gyroscope, 3axis accelerometer, and a Digital Motion Processor ${ }^{\mathrm{TM}}$ (DMP).The Honeywell's HMC5883L which is a 3-axis digital compass. The BMP085 is a high-accuracy chip to detect barometric pressure and temperature.

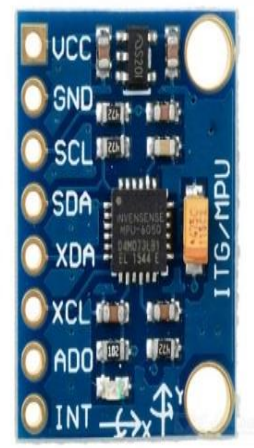

Figure 3. Gyro Sensor

\subsection{POLOLU - 75:1 Metal Gearmotor}

POLOLU -75:1 Metal Gearmotor is a powerful brushed DC motor with metal gearbox intended for operation at $12 \mathrm{~V}$. These units have a 16mm-long, 6mm-diameter D-shaped output shaft. This gearmotor is also available with intergrated encoder.

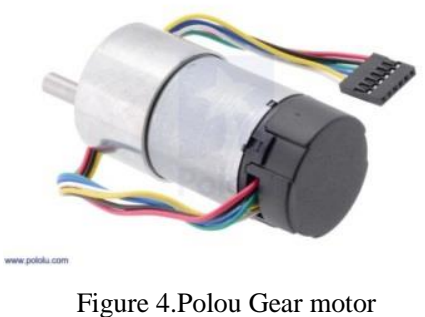

\subsection{Pin Connection of Purpose System}

The circuit diagram of the system is shown in Figure 5.

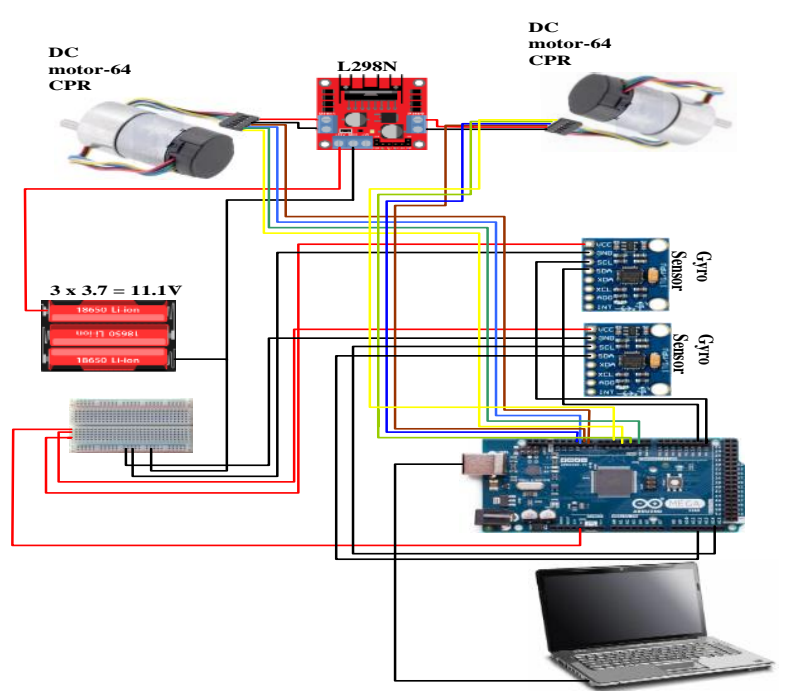

Figure 5. Connection of circuit diagram

\section{TEST AND RESULTS}

The Controller in any system plays very significant role and helps in taking remedial control action. The essential function of controller is to maintain the output at that level so that there is no difference between the measured output and set point value. PID controllers are the predominant type of controllers which are used in almost every process industry [3]. PID controller is widely used in industries due to its simplicity and easy to implement. A PID controller calculates an "error" and tries to minimize it by adjusting the control inputs. It improves the transient response of the system by reducing overshoot and by shortening the settling time and also has the ability to eliminate steady state offset through integral action. The transfer function for the PID controller can be written as,

$u(t)=M V(t)=K_{p} e(t)+K_{i} \int e(t) d t+K_{d} \frac{d e(t)}{t}$

Equivalently, the transfer function in the Laplace domain of the PID Controller

$L(s)=K_{P}+K_{i} / s+K_{d} s$

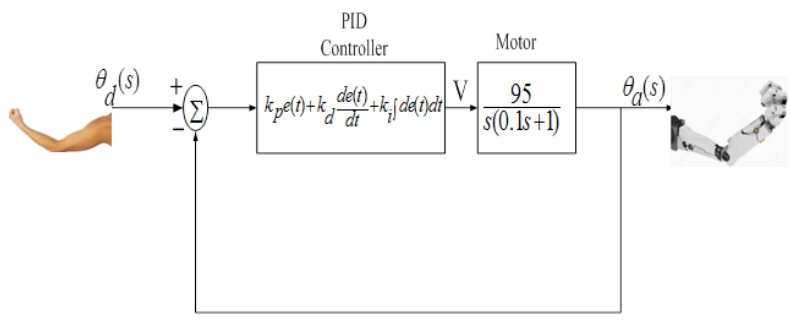

Figure 6. Diagram of PID Controller with motor

The Manual tuning method is used in the system. Because Manual tuning method can be relatively time consuming, particularly for system with long loop time. The various tuning steps of control gain is shown in Table 1. The system have been decided the gain result of $\mathrm{K}_{\mathrm{p}}=0.3, \mathrm{~K}_{\mathrm{i}}=0.01$ and $\mathrm{K}_{\mathrm{d}}=0.02$. Because the motor's actual angle is acceptably reached to its desired angle after a load disturbance.

Results, Steady State Error $=\mathrm{e}_{\mathrm{ss}}=0$, Rise Time $=\mathrm{t}_{\mathrm{r}}=0.3$. (no significant change), Setting Time $=\mathrm{t}_{\mathrm{s}}=0.5$ (no significant change) and slightly overshoot remained. 
Table 1. The Tuning Step of PID controller gains using Manual tuning Method

\begin{tabular}{|c|c|c|c|}
\hline Tuning Step & $\mathbf{K}_{\mathbf{p}}$ & $\mathbf{K}_{\mathbf{i}}$ & $\mathbf{K}_{\mathbf{d}}$ \\
\hline $1^{\text {st }}$ & 0.1 & 0 & 0 \\
\hline $2^{\text {nd }}$ & 0.2 & 0 & 0 \\
\hline $3^{\text {rd }}$ & 0.3 & 0 & 0 \\
\hline $4^{\text {th }}$ & 0.3 & 0.01 & 0 \\
\hline $5^{\text {th }}$ & 0.3 & 0.01 & 0.01 \\
\hline $6^{\text {th }}$ & 0.3 & 0.01 & 0.02 \\
\hline
\end{tabular}

The simulation result of PID controller using set point 90 is shown in Figure 7 and the simulation result of the system is shown in Figure 8 and the experiment test for motor position is shown in Figure 9.

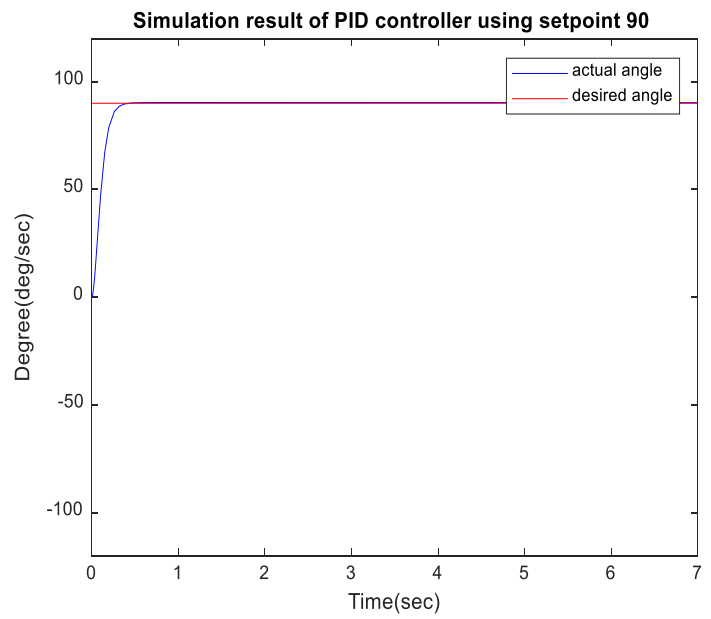

Figure 7. The Simulation result of PID Controller using set point 90

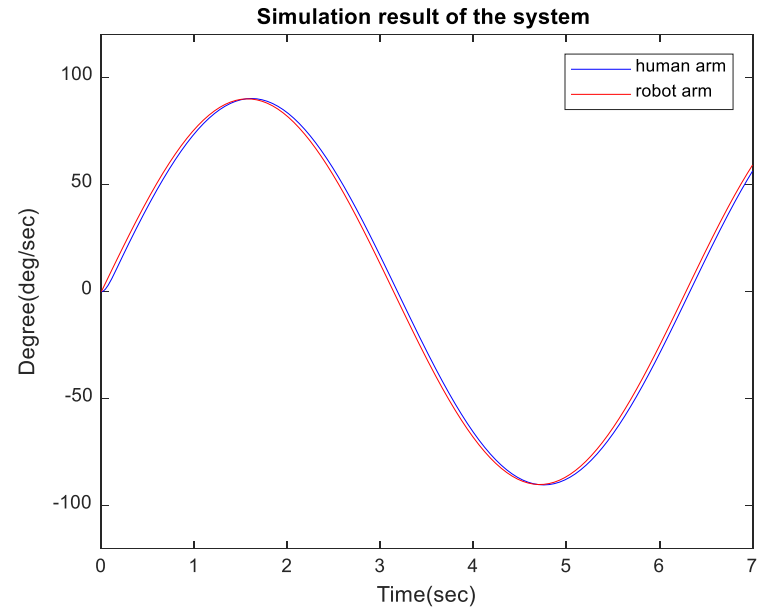

Figure 8. The Simulation result of Robot Arm Imitate Human Arm

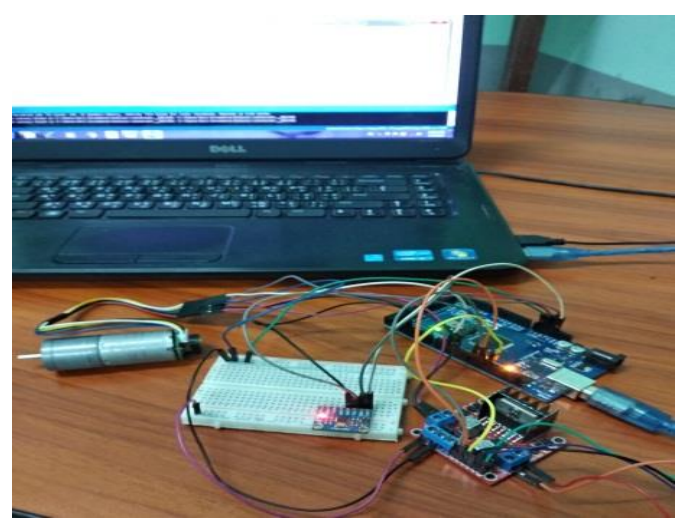

Figure 9. Experiment test for motor position .

The implementation of robot arm is shown in Figure 10 and the experiment test of robotic arm imitating human arm system is shown in figure 11 and the experiment result of overall system is shown in Figure 12.

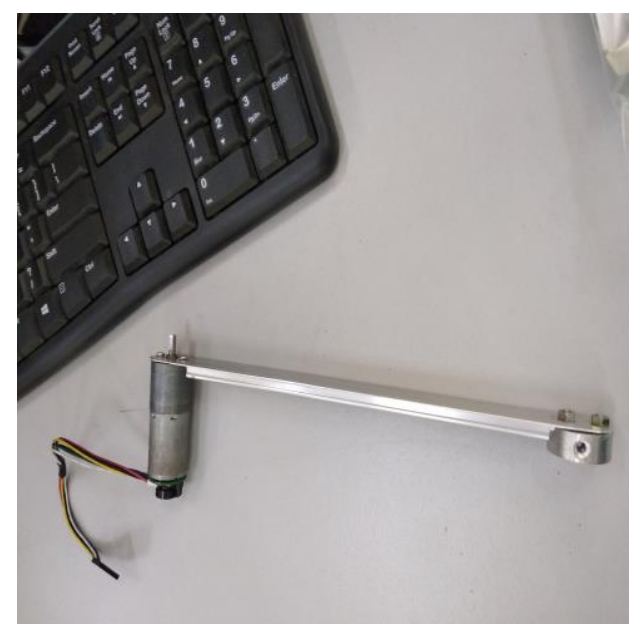

Figure 10. The implementation of robot arm

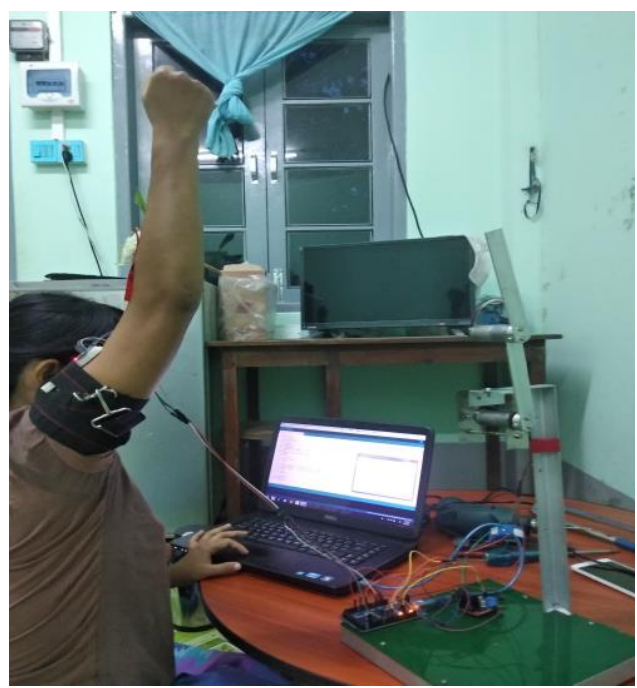

Figure 11. The experiment test of overall system 


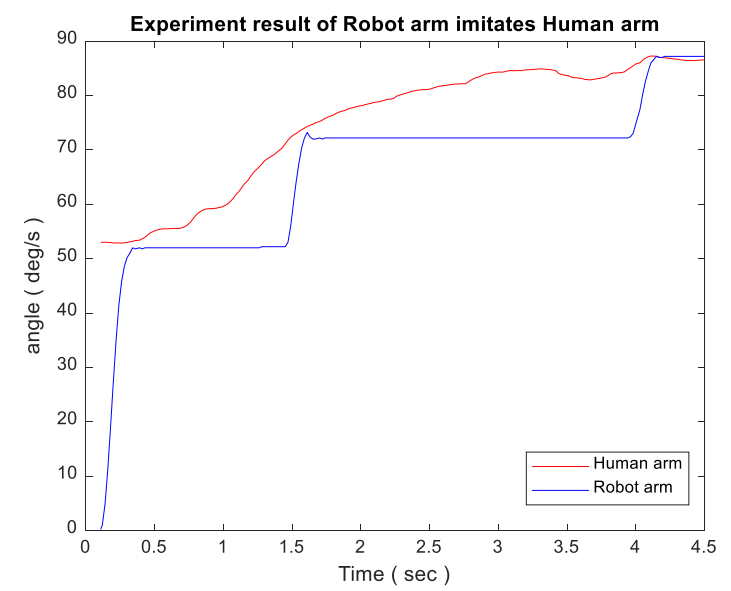

Figure 12 . The Experiment result of robot arm imitate human Arm

\section{FUTURE WORK}

In order to develop the efficiency of the robotic arm, the wireless sensors can be employed to enhance interface between the human arm and robotic arm [4]. Different sensors such as electromyography (EMG), accelometer, and airpressure sensors [5]-[6] can be incorporated; and corresponding algorithms can be investigated. In order to improve the response accuracy, the machine learning algorithms such as the artificial neural network (ANN) classifier and fuzzy logic classifier can be applied.

\section{CONCLUSION}

The system are going to develop is a device which is efficient , light weight, comprising less machinery, and has applications in various domestic and industrial purposes. According to the result, the robot action is similar as $98 \%$ accuracy to human arm action. From observation that has been made, it clearly shows that its movement is precise, accurate, and is easy to control and user friendly to use. This robotic arm control method is expected to overcome the problem such as placing or picking object that away from the user, pick and place hazardous object in a very fast and easy manner.

\section{ACKNOWLEDGEMENTS}

Firstly, the author would like to acknowledge Dr. Thein Gi, Rector of Technological University (Thanlyin), for her kind permission to carry out this research work. The authors would like to thank many colleagues from digital image processing research group of Department of Electronic Engineering of Technological University (Thanlyin). The author particularly wishes to acknowledge all the teachers from Department of Electronic Engineering, Technological University (Thanlyin), for their support, encouragement and invaluable guidance in preparation of this research. The authors would like to express their thanks to all persons who have given support during the preparation period of this research work.

\section{REFERENCES}

[1] Rajesh Kannan Megalingam, Sricharan Boddupalli, K G S Apuroop K . 2017. "Robotic Arm Control through Mimicking of Miniature Robotic arm”, 2017 International Conference on Advanced Computing and Communication Systems (ICACCS -2017).
[2] Pankaj S Lengare, Milind E Rane" Human hand TRACKING USING MATLAB TO CONTROL ARDUINO BASED ROBOTIC ARM", 2015 INTERNATIONAL CONFERENCE ON Pervasive COMPUTING (ICPC).

[3] N.S. Rathore, D.P.S. Chauhan, V.P. Singh. 2015. “ Tuning of PID Controller for Position Control of DC Servo Motor using Luus-Jaakola Optimization", IEEE International Conference on Computer, Communication and Control (IC4- 2015).

[4] R. Sekhar, R. Musalay, Y. Krishnamurthy, B. Shreenivas. 2012. "Inertial sensor based wireless control of a robotic arm," IEEE International Conference on Emerging Signal Processing Applications, pp. 87-90, Las Vegas, NV.

[5] M. Georgi, C. Amma, T. Schultz. 2015. "Recognizing hand and fingergestures with IMU based motion and EMG based muscle activity sensing," Proceedings of the International Conference on Bioinspired Systems and Signal Processing, pp. 99- 108, Lisbon, Portugal.

[6] P. Jung, G. Lim, S. Kim, K. Kong. 2015. “A wearable gesture recognition device for detecting muscular activities based on air-pressure sensors," IEEE Transactions on Industrial Informatics, 11(2), pp. 485494. 